\title{
Differential neural response to positive and negative feedback in planning and guessing tasks
}

\author{
R. ELLIOTT, ${ }_{\S}$ C. D. FRITH*† and R. J. DOLAN*‡ \\ *Wellcome Department of Cognitive Neurology, Institute of Neurology, 12 Queen Square, London WC1N 3BG U.K.; \\ $\dagger$ University College London, Gower Street, London WC1E 6BT, U.K.; $;$ Royal Free Hospital School of Medicine, \\ Rowland Hill Street, London NW3 2PF, U.K.
}

(Received 16 October 1996; accepted 14 March 1997)

\begin{abstract}
The neural mechanisms by which emotional and cognitive processing interact are unknown. Evidence from animal studies and neurological patients suggests that regions of the ventral striatum and orbitofrontal cortex, together with limbic structures such as the amygdala, are critical to such interactions. We used positron emission tomography to study the neural systems engaged by processing performance feedback under two conditions involving either a complex cognitive or a matched guessing task. The main activations associated with the processing of performance feedback under different task conditions involved foci in the medial caudate nucleus and the ventromedial orbitofrontal cortex. A differential modulation of these activations as a function of task type was observed. In particular the orbitofrontal activation associated with the presence of feedback was only seen in the guessing task. These data suggest that the ventral striatum and orbitofrontal cortex are involved in processing of feedback information, findings consistent with animal and neurological studies. We propose that differential activation associated with guessing compared to planning suggests enhanced neural processing of feedback when the outcome of a task is uncontrollable or when information must be assimilated across a number of trials to assess performance. $C 1997$ Elsevier Science Ltd
\end{abstract}

Key Words: PET-reward; orbitofrontal cortex; ventral striatum.

\section{Introduction}

In many laboratory tests of cognition, subjects are given feedback information evaluating their performance. Cognitive performance is therefore confounded by emotive and evaluative processes involved in responding to this feedback. The laboratory situation provides a simplistic model for real life where behaviour is susceptible to extrinsic or intrinsic reward or the avoidance of punishment. The impact of even abstract forms of reward is amply demonstrated by the popularity of computer games; players strive for 'success' in spite of the absence of external consequences of high performance levels.

Functional imaging studies of cognitive activation have almost invariably failed to focus on emotive and evaluative aspects of cognition, confining themselves to a determination of the neural correlates of cognitive processes and subprocesses. There have been studies concentrating on specific emotional processing; for example processing of and memory for emotional pictures $[19,10]$

$\S$ Correspondence: tel: $+44(0171) 8337485$; fax: $+44(0171)$ 813 1420; e-mail: r.elliott (affil.ion.ucl.ac.uk. or processing of facial expressions [15, 22]. However, in the context of neuroimaging few studies have addressed the influence of evaluative or emotive processes on cognitive performance in normal subjects. One aspect which has been studied is the controllability of a cognitive task. Schneider $e t a l$. [26] studied regional cerebral blood flow ( $\mathrm{rCBF}$ ) associated with solvable and insoluble anagrams and found reciprocal diencephalic and limbic activations. This study in effect considered implicit failure on a complex task. Our study aimed to address success and failure more explicitly by manipulating the level of performance feedback on a cognitive task. Feedback can be used to guide and monitor performance, with subjects adjusting their approach to the task in the light of feedback information. However, there is also a more purely emotional influence of feedback due to the motivational consequences of success and failure.

The neural substrates of reward and punishment have been widely studied in experimental animals. Various brain regions have been associated with different aspects of this type of feedback processing. The amygdala has been shown to be involved in the association of stimuli with primary and secondary reinforcers in reward-related learning tasks $[13,14]$. The amygdala has strong ana- 
tomical connections with the ventral striatum [25], an area also implicated in the response to reward. For example, Schultz et al. [27] demonstrated that ventral striatum neurons are activated before the expected delivery of reward and on this basis, argued that ventral striatal neurons have access to central representations of reward. Thus, unlike the dorsal striatum, the ventral striatum is predominantly concerned with processing reward related information and has been proposed to contribute to motivational aspects of behavioural output.

Another region with access to central representations of reward-related information is the orbitofrontal cortex, which has strong connections with the ventral striatum [28]. The response of neurons in the monkey orbitofrontal cortex to sensory stimuli are also dependent on the meaning of the stimuli [32]. This region also has neurons that show activity after a behavioural response and such neurons could code the outcome of a trial. Thorpe et al. [32] therefore suggested that the orbitofrontal cortex can influence the reinforcement properties of stimuli and can rapidly moderate this influence in the light of recent experience.

In animal studies, feedback takes the form of either rewards or punishments that are directly reinforcing at a behavioural level. In man, it is clear that less direct forms of feedback are also highly effective. People are motivated to succeed in tasks even when the sole reward is the knowledge that they have succeeded. Studies of neurological patients have provided clear parallels with the animal literature. Rolls et al. [24] studied a group of patients with ventral frontal lesions and found severe impairments relative to a group with non-ventral prefrontal lesions in the reversal and extinction of simple visual discrimination tasks. Remarkably, in this study patients could report verbally that reinforcement contingencies had changed but could not modify their behaviour appropriately. This is consistent with the effects of orbitofrontal lesions in monkeys on performance of analogous tasks $[6,9,17,18]$. The deficits reported by Rolls et al. [24] were highly specific, the same group of patients had normal IQ and verbal memory and could perform higher cognitive tasks such as the Tower of London planning task [30].

Evidence from studies with animals and with neurological patients suggests that an emotional component of processing can be, at least partially, separated from direct task performance related processing. This emotional component is an important aspect of many cognitive processes where emotively charged feedback information can be used to guide and modify performance. The present study aimed to redress this by treating the level of performance feedback as an experimental variable. The experimental tasks were a variation of the one-touch planning task used in a previous PET study [2] and a guessing task matched for perceptuomotor components. Both tasks were performed with three levels of feedback; positive, negative and no feedback. We aimed to test the hypothesis that the presence of feedback would be associated with activation in structures in a limbic-striatal-orbitofrontal system. We further predicted that activity in this network would be modulated by task-type with greater feedback-related activation in the planning task where the feedback informs the subject about how well they are performing a cognitive operation.

\section{Methods}

\section{Subjects}

Six right-handed male volunteers aged between 27 and 50 were recruited for this study. Subjects with a neurological or psychiatric history were excluded. The study was approved by the local hospital ethics committee, and permission to administer radioactive substances was obtained from the Administration of Radioactive Substances Advisory Committee (ARSAC) U.K. Informed written consent was obtained prior to the study.

\section{Cognitive activation paradigm}

The design of this experiment was a two by three factorial design with experimental task (two levels; planning and guessing). as one factor and feedback condition (three levels; positive, negative and no feedback) as the other.

\section{Planning task}

This task was based on the Tower of London task [30], using the one-touch approach developed by Owen et al. [23]. A version of this task has been used in a previous PET study [2] and the present version was a variant of this paradigm. Subjects were presented two arrays of coloured balls and asked to work out the minimum number of moves needed to transform one array to the other, according to rules explained prior to scanning. They were given $10 \mathrm{sec}$ in which to perform this task, after which the arrays disappeared and subjects had to press the one of six labelled buttons which corresponded to their answer. Problems were all of three moves or harder and subjects typically require more than $10 \mathrm{sec}$ to solve problems of this difficulty $[2,23]$. Imposition of the time limit therefore prevented subjects being certain of their answers and therefore rendered them more likely to believe false feedback.

Subjects were presented with a series of 10 unique problems during each scan. The sequence started 1 min before scanning to ensure the establishment of the appropriate cognitive set. Subjects were explicitly told prior to each series that they should aim to optimise performance.

\section{Guessing task}

In this task, subjects were presented with two identical arrays of coloured balls and asked to watch them until they disappeared (after $10 \mathrm{sec}$ ), at which point they should immediately press any one of the six response buttons. They were told that on each trial, three of the buttons would be randomly assigned as correct. It was stressed to subjects before each sequence that this assignment was purely chance such that each response constituted a 50:50 guess. Subjects were again presented with a series of 10 problems, starting one minute before scanning. 


\section{Feedback conditions}

Each of the two tasks above was performed under three feedback conditions. Feedback was given after each trial and took the form of a large tick and the words "YOU ARE RIGHT" or a large cross and the words "YOU ARE WRONG", presented on the screen for one second immediately following each response. In one condition, $100 \%$ of trials were followed by positive feedback, regardless of whether or not the responses made were correct. In another condition $80 \%$ of trials were followed by negative feedback and $20 \%$ by positive, regardless of whether the responses were actually correct. In the third condition, no feedback was given; between trials subjects saw a display comprising a large triangle and the words "PLEASE WAIT".

There were thus six conditions in all and subjects performed each condition twice. Conditions were fully counterbalanced within and between subjects.

\section{PET scanning technique}

Regional cerebral blood flow was measured with an ECAT $\mathrm{HR}+$ scanning system. For each scan, $555 \mathrm{Mbq}$ of $\mathrm{H}_{2}{ }^{15} \mathrm{O}$. were flushed through a venous cannula in the left antecubital vein with normal saline over $20 \mathrm{sec}$ at a rate of $10 \mathrm{ml} / \mathrm{min}$ by an automatic pump. After a delay of about $35 \mathrm{sec}$, a rise in counts could be detected at the head, peaking $30-40 \mathrm{sec}$ later, varying for individual subjects. The data were acquired during one $90 \mathrm{sec}$ frame, beginning $5 \mathrm{sec}$ before the rising phase of the head curve. A total of 12 scans were performed at intervals of 8 min. Correction for attenuation was made by performing a transmission scan with an exposed $68 \mathrm{Ge} / 68 \mathrm{Ga}$ external ring source before each session.

Images were reconstructed by filtered back projection to give a resolution of $6.0 \mathrm{~mm}$ at full width half maximum and displayed in a $128 \times 128$-pixel format with 63 planes rendering the voxels approximately cubic.

\section{Data analysis}

Data were analysed using Statistical Parametric Mapping (SPM95, Wellcome Department of Cognitive Neurology, London, U.K.; [12]). Scans were realigned using the first as a reference and were subsequently transformed into a standard space corresponding to the stereotactic atlas of Talairach and Tournoux [31]. These normalised images were smoothed with a 16-mm FWHM isotropic Gaussian kernel.

Conditions for each subject were specified in the appropriate design matrix which also included global activity as a confounding covariate and can therefore be considered an ANCOVA. Effects at each and every voxel were estimated according to the general linear model and regionally specific effects were compared using linear contrasts. The resulting set of voxel values for each contrast constituted a statistical parametric map of the $t$ statistic (SPM $\{t\}$ ) which was then transformed to the unit normal distribution, $\operatorname{SPM}\{Z\}$.

\section{Results}

\section{Performance data}

When subjects were debriefed after scanning, all claimed to have realised that the feedback was irrelevant to performance on both the planning and guessing tasks approximately half way through the 12 scans. Figure 1 shows the performance of subjects on the planning task in the different feedback conditions. It is clear from this figure that performance overall (grand mean $74 \%$ correct) was substantially above chance level $(17 \%)$ and in fact is only slightly worse than performance in the study of Baker et al. [2] where there was no time constraint (grand mean $81 \%$ correct). These data show that subjects were engaged in relatively efficient planning, in spite of the imposed time limit. This is confirmed by the difference in overall response latencies for the planning and guessing tasks ( $830 \mathrm{msec}$ for planning; $420 \mathrm{msec}$ for guessing). As Fig. 1 shows, subjects performed less accurately in the negative feedback condition than the positive or neutral conditions, even on the second presentation when they claimed to be aware the feedback was irrelevant. This strongly suggests that even when subjects were aware of the invalidity of the feedback, it still carried affective salience.

\section{$r C B F$ changes related to the comparison of the planning and guessing tasks}

This comparison represents the main effects of the cognitive task across all feedback conditions (Table 1).

\section{Increases in $\mathrm{CBF}$ associated with planning compared with guessing}

Significant activations $(P<0.001)$ were observed in the medial occipital cortex bilaterally (BA 19), the cuneus

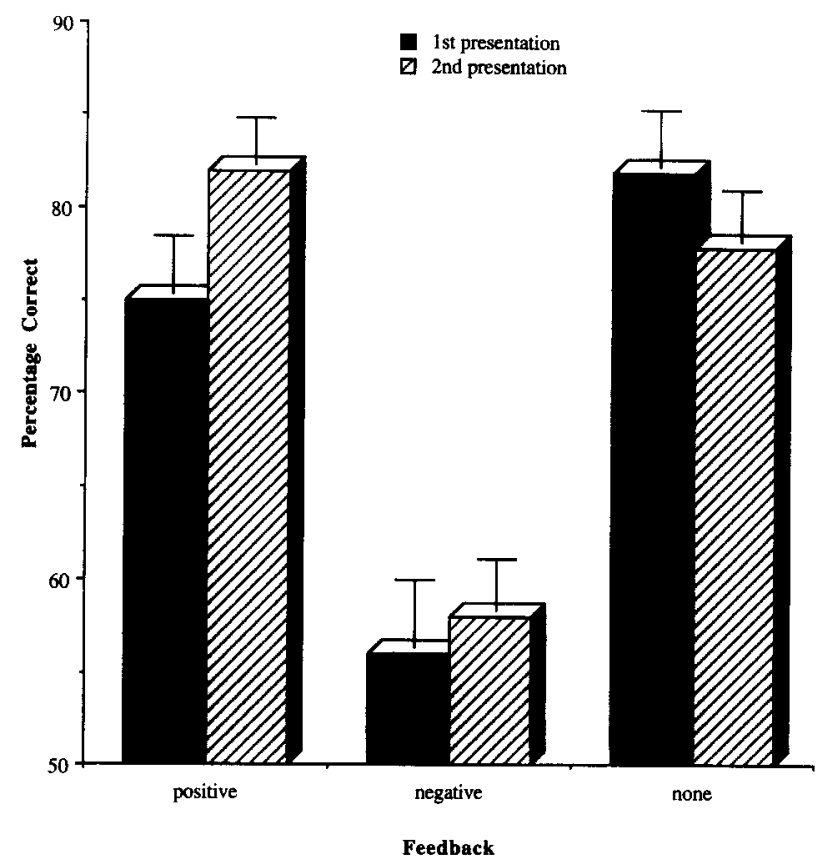

Fig. 1. Cognitive performance of the subjects on the planning task under the three feedback conditions. Mean percentage of problems solved correctly is shown. Bars show standard errors of the mean. Note that each condition was performed twice (Ist and 2 nd presentation) in a counterbalanced order. 
Table 1. Co-ordinates of maximal significant changes in rCBF in planning compared to guessing

\begin{tabular}{|c|c|c|c|c|c|c|}
\hline \multirow[b]{2}{*}{ Region of activation } & \multirow[b]{2}{*}{ Left/ right } & \multirow{2}{*}{$\begin{array}{l}\text { Brodmann's } \\
\text { area }\end{array}$} & \multicolumn{3}{|c|}{ Talairach co-ordinates } & \multirow{2}{*}{$\begin{array}{c}Z \text { value }(3.09 \text { for } \\
P<0.001)\end{array}$} \\
\hline & & & $x$ & $y$ & $z$ & \\
\hline \multicolumn{7}{|c|}{ rCBF increases } \\
\hline \multirow[t]{2}{*}{ Medial occipital cortex } & $\mathrm{L}$ & 19 & -28 & -84 & 16 & 6.78 \\
\hline & $\mathrm{R}$ & 19 & 32 & -84 & 16 & 6.88 \\
\hline Cuneus & $\mathrm{R}$ & 17 & 6 & -98 & 4 & 3.42 \\
\hline \multirow[t]{2}{*}{ Premotor cortex } & $\mathrm{L}$ & 6 & -28 & -2 & 52 & 4.36 \\
\hline & $\mathrm{R}$ & 6 & 28 & 0 & 48 & 4.30 \\
\hline \multirow[t]{2}{*}{ Orbitofrontal gyrus } & $\mathrm{L}$ & 47 & -20 & 26 & -24 & 4.25 \\
\hline & $\mathrm{R}$ & 47 & 20 & 28 & -24 & 3.17 \\
\hline Anterior insula & $\mathrm{R}$ & 45 & 28 & 20 & 4 & 3.21 \\
\hline Thalamus & $\mathrm{R}$ & & 12 & -14 & 12 & 3.30 \\
\hline Putamen & $\mathrm{R}$ & & 20 & 14 & 4 & 3.15 \\
\hline \multicolumn{7}{|c|}{ rCBF decreases } \\
\hline \multirow[t]{2}{*}{ Medial temporal gyrus } & $\mathbf{R}$ & 21 & 44 & -12 & -4 & 6.55 \\
\hline & $\mathrm{L}$ & & -54 & -20 & -12 & 6.61 \\
\hline Anterior frontal cortex & $\mathrm{R}$ & 10 & 6 & 52 & 12 & 6.63 \\
\hline Postcentral gyrus & $\mathrm{R}$ & 4 & 26 & -32 & 52 & 3.52 \\
\hline Inferior frontal gyrus & $\mathrm{R}$ & 45 & 44 & 32 & 8 & 3.19 \\
\hline
\end{tabular}

(BA 17), the premotor cortex bilaterally (BA 6), the orbitofrontal gyri bilaterally (BA 47) and the right anterior insula (BA 45). Subcortical activations at a similar level of significance were also observed in the right thalamus and right putamen. This network was less widespread than that observed by Baker et al. [2]; in particular activations were not seen in the dorsolateral and rostrolateral prefrontal cortices or the anterior cingulate. However, in the present study the control task was more demanding, requiring subjects to make a guess rather than pressing a predetermined key. This control task therefore involves more active processing, including response selection which is associated with activations in frontal regions.

\section{Decreases in $r C B F$ associated with planning compared with guessing}

Significant $(P<0.001)$ rCBF decreases were observed bilaterally in the medial temporal gyri (BA 21) and in the right postcentral gyrus (BA 4) and anterior frontal and inferior frontal gyri (BA 10, BA 45, respectively).

\section{$r C B F$ changes related to the nature of performance feed- back}

These comparisons represent main effects of feedback across the two task conditions, see Table 2, Fig. 2.

Changes in $\mathrm{CCBF}$ associated with presence compared with absence of feedback

In this comparison the two feedback present conditions (positive and negative) were averaged and compared with the no feedback condition. Significant activations $(P<0.001)$ were seen in the medial caudate nuclei bilaterally, the right superior temporal gyrus and on the left close to the depths of the sulcus connecting the medial and superior temporal gyri. When adjusted blood flow values in the medial caudate nucleus focus were studied, they were seen to be very similar in the positive and negative feedback conditions and lower in the no feedback condition, see Fig. 2(b). No significant decreases in rCBF were seen.

\section{Changes in $\mathrm{CCBF}$ associated with positive compared with absence of feedback}

The pattern of activation was identical to that described in the preceding comparison.

\section{Changes in $\mathrm{CCBF}$ associated with negative compared with no feedback}

The pattern of activation was again similar to that in the previous comparisons but the superior temporal gyrus activation was not significant and the other activations were less extensive and less significant.

Changes in $\mathrm{C} C \mathrm{BF}$ associated with positive compared with negative feedback

Significant $(P<0.001)$ increases in rCBF were seen in the right fusiform gyrus (BA 20), the left postcentral gyrus (BA 40) and the thalamus. No significant decreases were seen. 
Table 2. Coordinates of maximal significant change in rCBF associated with the presence compared to the absence of performance feedback

\begin{tabular}{|c|c|c|c|c|c|c|}
\hline \multirow[b]{2}{*}{ Region of activation } & \multirow[b]{2}{*}{ Left/right } & \multirow{2}{*}{$\begin{array}{l}\text { Brodmann's } \\
\text { area }\end{array}$} & \multicolumn{3}{|c|}{ Talairach co-ordinates } & \multirow{2}{*}{$\begin{array}{c}Z \text { value }(3.09 \text { for } \\
P<0.001)\end{array}$} \\
\hline & & & $x$ & $y$ & $z$ & \\
\hline \multicolumn{7}{|c|}{ rCBF increases } \\
\hline \multirow[t]{2}{*}{ Medial caudate nucleus } & $\mathrm{R}$ & & 8 & 16 & 16 & 3.67 \\
\hline & $\mathrm{L}$ & & -10 & 16 & 4 & 3.56 \\
\hline \multirow{2}{*}{ Superior temporal gyrus } & $\mathrm{L}$ & 22 & -32 & -34 & 4 & 3.72 \\
\hline & $\mathrm{R}$ & 38 & 42 & 8 & -8 & 3.25 \\
\hline
\end{tabular}

(a)
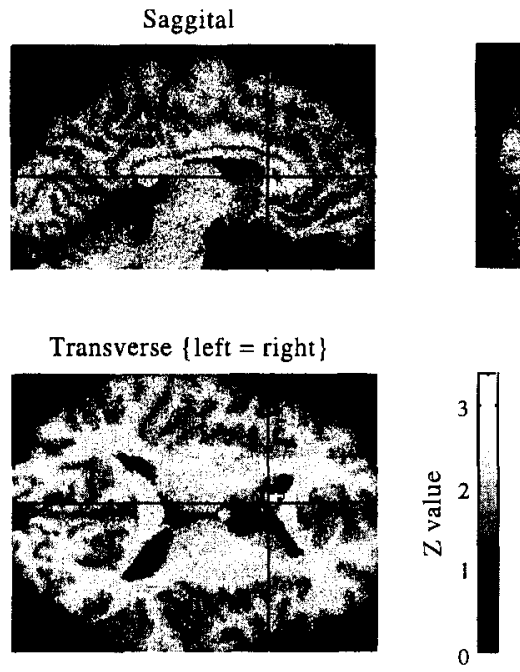

(b)

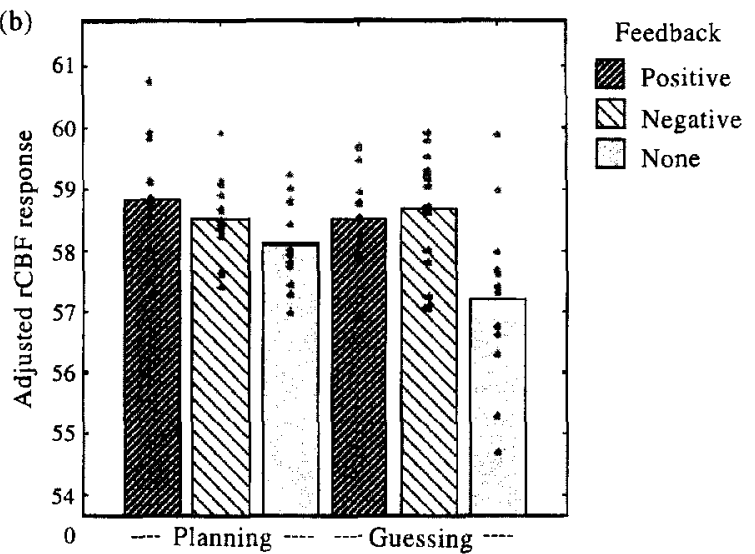

Fig. 2. rCBF increases associated with the presence compared to the absence of feedback. (a) The rCBF rendered onto a standard MRI template and focused on the medial caudate nucleus activation. (b) Adjusted values of blood flow under the six experimental conditions in the medial caudate nucleus.

$r C B F$ changes related to the modulation of task performance by different types of feedback

These comparisons represent interaction terms in a factorial analysis. The main effects described above suggest that the effects of positive and negative feedback are qualitatively similar and since the same was true of the interaction terms, only the comparisons of averaged positive and negative feedback with no feedback are described in the interests of clarity, see Table 3 and Fig. 3.
Increases in $r C B F$ in feedback compared to no feedback conditions in planning compared to guessing tasks

Significantly $(P<0.001)$ increased activation was seen in the left occipital cortex (BA 19) and the left percentral gyrus (BA 4).

Increases in $r C B F$ in feedback compared to no feedback conditions in guessing compared to planning tasks

Significantly $(P<0.001)$ increased activation was seen in the ventromedial orbitofrontal cortex (BA 25), the anterior frontal cortex (BA 10) and the left inferior frontal gyrus (BA 47). When adjusted blood flow values in the orbitofrontal cortex focus were considered, this interaction was shown to be due to greater blood flow in the feedback than the no feedback condition in the guessing task rather than lower blood flow in the planning task. In other words, the raw data indicate that this interaction represents a true increase in $\mathrm{rCBF}$ associated with feedback during guessing.

$r C B F$ changes related to feedback in the planning task and the guessing task

The significant interaction terms described above suggested that the comparisons representing simple main effects of feedback in the two tasks should be considered separately, see Table 4 and Fig. 4.

Changes in $r C B F$ associated with the presence of feedback in the planning task

There were no significantly increased activations associated with the presence compared to the absence of feedback in the planning task. Significant decreases in activation $(P<0.001)$ were observed in the left postcentral gyrus (BA 40) and the right putamen. 
Table 3. Coordinates of maximal significant change in rCBF associated with the modulation of planning compared to guessing performance by presence compared to absence of feedback

\begin{tabular}{lcccccc}
\hline & \multicolumn{2}{c}{ Brodmann's } & \multicolumn{2}{c}{ Talairach co-ordinates } & \multicolumn{2}{c}{$Z$ value (3.09 for } \\
Region of activation & Left/ right & area & $x$ & $y$ & $z$ & $P<0.001)$ \\
\hline & \multicolumn{7}{c}{ rCBF increases } \\
Occipital cortex & $\mathrm{L}$ & 19 & -20 & -88 & 28 & 3.81 \\
Precentral gyrus & $\mathrm{L}$ & 4 & -18 & -24 & 68 & 3.28 \\
& & rCBF decreases & & & & \\
Ventromedial orbitofrontal cortex & $\mathrm{R}$ & 25 & 2 & 26 & -10 & 3.61 \\
Anterior frontal cortex & $\mathrm{R}$ & 10 & 14 & 58 & -4 & 3.35 \\
Inferior frontal gyrus & $\mathrm{L}$ & 47 & -28 & 22 & 0 & 4.05 \\
\hline
\end{tabular}

(a)
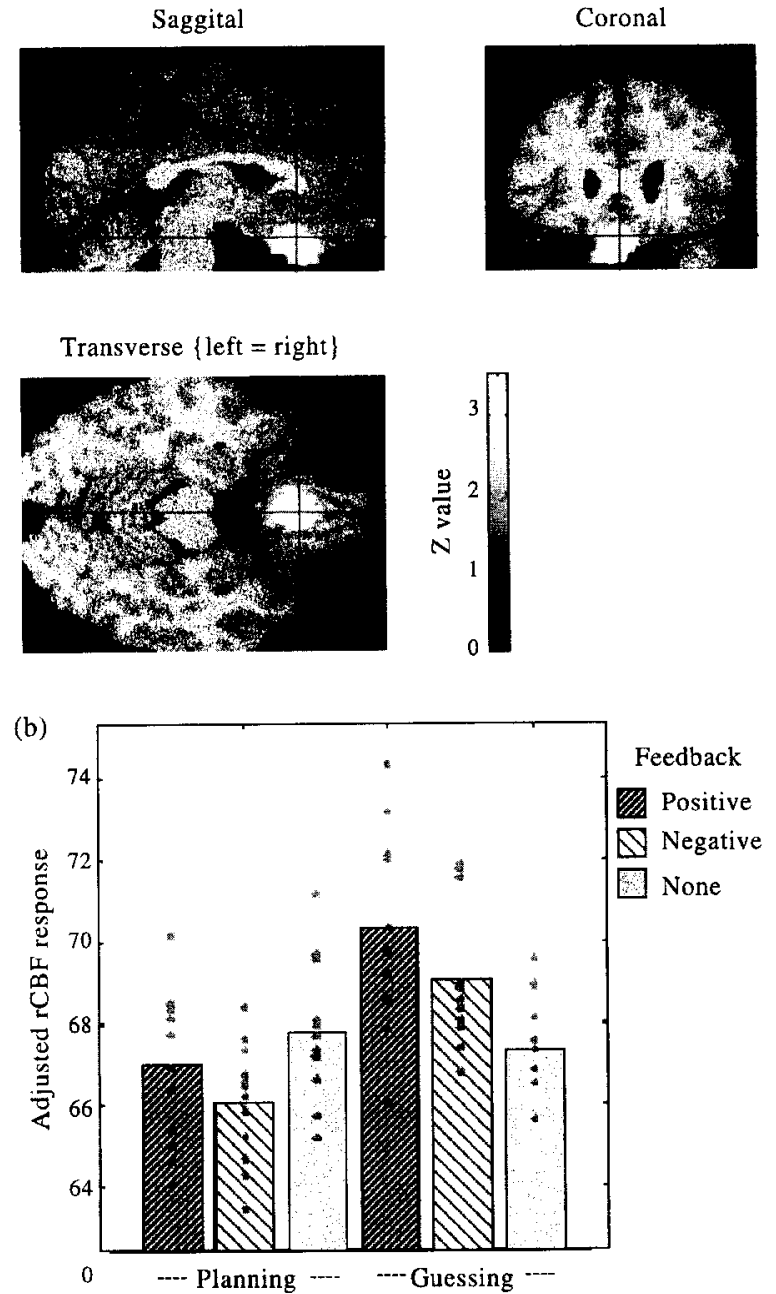

Fig. 3. rCBF increases associated with the modulation of guessing compared to planning by presence compared to the absence of feedback, i.e those areas more activated by the presence of feedback in the guessing task than in the planning task. (a) The rCBF rendered onto a standard MRI template and focused on the ventromedial orbitofrontal activation. (b) Adjusted values of blood flow under the six experimental conditions in the ventromedial orbitofrontal cortex.

Changes in $r C B F$ associated with the presence of feedback in the guessing task

Significant $(P<0.001)$ increases in activation were observed in the medial caudate nucleus extending rostrally to the ventral orbitofrontal cortex, in the left inferior frontal gyrus (BA 47) and the right superior temporal gyrus (BA 38). In both the medial caudate and the orbitofrontal cortex foci, blood flow values were higher in the feedback conditions in the guessing task than in the planning task and lower in the corresponding no feedback condition. This suggests an exaggerated response to presence and absence of feedback in these regions in the guessing task compared to the planning task. No significant decreases were observed.

\section{Discussion}

The main findings of this study were activations of the medial caudate and orbitofrontal cortex associated with the presence compared to the absence of feedback under different task conditions. The medial caudate activation was seen across planning and guessing tasks but this activation, and also the orbitofrontal activation, was modulated by task type as shown by the interaction term of the analysis. In both structures, differential activation in the presence relative to the absence of feedback was greater in the guessing than the planning task however consideration of adjusted blood flow values showed that this interaction represented different effects in the medial caudate and orbitofrontal foci. Adjusted values of regional cerebral blood flow in the medial caudate showed that it was similarly activated in the presence of feedback in both tasks, however activation in the feedback absent condition was significantly lower in the guessing than the planning task. In the orbitofrontal cortex of the network, there was no enhanced neural response to the presence of feedback in the planning task (if anything, blood flow was lower in the feedback conditions), while in the guessing task, differential orbitofrontal activation in response to feedback was seen. Thus, adjusted orbitofrontal blood flow was higher when feedback was present in the guessing compared to the planning task with no difference when feedback was absent.

Our findings therefore confirmed the hypothesis that performance feedback would be associated with differential activations in regions including the ventral striatum 
Table 4. Coordinates of maximal significant change in $\mathrm{rCBF}$ associated with presence compared to absence of feedback in the guessing task

\begin{tabular}{|c|c|c|c|c|c|c|}
\hline \multirow[b]{2}{*}{ Region of activation } & \multirow[b]{2}{*}{ Left/right } & \multirow{2}{*}{$\begin{array}{l}\text { Brodmann's } \\
\text { area }\end{array}$} & \multicolumn{3}{|c|}{ Talairach co-ordinates } & \multirow{2}{*}{$\begin{array}{c}Z \text { value }(3.09 \text { for } \\
P<0.001)\end{array}$} \\
\hline & & & $x$ & $y$ & $z$ & \\
\hline \multicolumn{7}{|c|}{ rCBF increases } \\
\hline Ventromedial orbitofrontal cortex & $\mathrm{R}$ & 11 & 10 & 30 & -20 & 3.64 \\
\hline Medial caudate nucleus & $\mathrm{R}$ & & 8 & 20 & 8 & 4.08 \\
\hline Inferior frontal gyrus & $\mathrm{L}$ & 47 & -22 & 22 & 0 & 3.96 \\
\hline Superior temporal gyrus & $\mathrm{R}$ & 38 & 40 & 8 & -12 & 3.53 \\
\hline
\end{tabular}
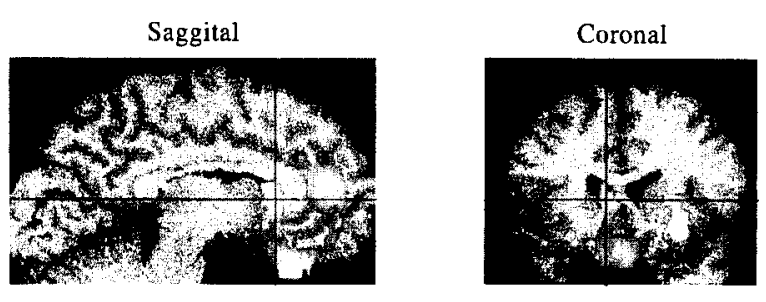

Transverse $\{$ left $=$ right $\}$
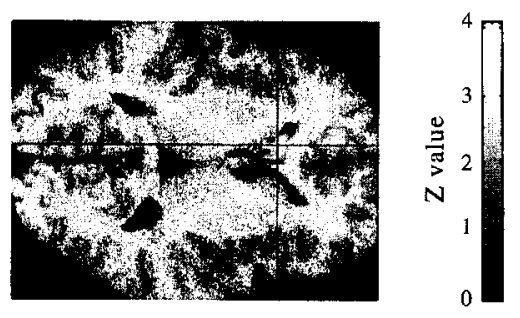

Fig. 4. rCBF increases associated with the presence compared to the absence of feedback in the guessing task, rendered onto a standard MRI template and showing the activations in the medial caudate and orbitofrontal cortex.

and orbitofrontal cortex. However, in both the medial caudate and the ventromedial orbitofrontal cortex, the magnitude of the difference between feedback and no feedback conditions was greater in the guessing than the planning task. This contradicted our a priori hypothesis that stronger activation would be seen in the planning than the guessing task. The fact that the orbitofrontal component of this network is activated during guessing but not planning is at first sight somewhat counter-intuitive. It might be expected that the response to performance feedback should be stronger in a task where subjects can generate an answer, since feedback in this case would inform them about their ability to solve the task. In a guessing task, a right or wrong response tells subjects nothing about their ability, and feedback might therefore be expected to be less salient.

The performance data indicate clearly that subjects were actually planning during the Tower paradigm rather than resorting to guesswork due to the time constraint. Further, the poorer performance in the negative feedback condition of the task suggests that even when subjects claimed to be aware that the feedback was false, it still influenced their performance. This result is compatible with accounts of affective responses to stimuli occurring prior to cognitive processing [34] and indicates that the feedback may have continued to have emotional content even when subjects had realised it was inaccurate. Additional support for this argument came from analysing the scan data to compare activations associated with conditions for the first presentation (when subjects were generally unaware of the invalidity of the feedback) and second presentation (when subjects had become aware). This analysis showed no differences between the activations in the critical structures of the ventral striatum and orbitofrontal cortex.

The apparently anomalous results become clearer when the cognitive requirements of the planning and guessing tasks are examined. In the planning task subjects do not need to process the feedback information as fully. When a task is soluble (as the planning task is), subjects presumably have some idea of whether their solution is correct before external feedback is given and therefore external feedback is less salient. In the guessing task by contrast, subjects are reliant on the external feedback to provide an assessment of performance. External feedback clearly influences planning performance, in that subjects solved fewer problems correctly in negative feedback conditions (see Fig. 1), an effect seen even when subjects claimed to have disregarded the feedback as false. Therefore the differential modulation of the neural response to feedback by task type is unlikely to be due to subjects not processing feedback information in the planning task. It is, however, possible that feedback is processed less deeply since immediately after the feedback a new problem is presented which engages subjects' attention and effort. This complex cognitive activity may inhibit further processing of the feedback. In the guessing task, the presentation of a new problem requires passive viewing for the first $10 \mathrm{sec}$ which is likely to interfere less with feedback processing. If subjects are generating feedback internally during the planning task, then the one condition where there is no feedback, either internal or external, is the 'no feedback' condition of the guessing task. Notably, blood flow within the caudate nuclei is at its lowest in this condition (see Fig. 2b), suggesting that the caudate activation is associated with processing of stimulus-reward associations. Activation of the caudate by internally generated feedback is compatible with the observation of Schultz et al. [27]. that neurons in the ventral striatum of monkeys are activated before the expected delivery of reward.

A related explanation for the observed differences lies 
in the perceived controllability of the task. In the planning task subjects believe that by modifying their performance they can increase their chances of reward; they have control over the type of feedback they receive. By contrast, in the guessing task it is stressed that they have no control and the feedback they receive is dependent on luck alone. This difference in controllability can be compared to the phenomenon of learned helplessness [29]. When animals are punished in a situation where they can learn to control the punishment, their behaviour develops differently to that of animals who receive yoked amounts of punishment but with no control. Animals with no control exhibit cringing and whimpering ('learned helplessness') compared to the more aggressive and active behaviour of the animals with control. The behaviour developed in response to negative feedback varies depending on the extent to which the situation can be controlled. The findings presented here may represent neural correlates of such an effect.

A further possible account of these findings, which is not incompatible with the previous explanation, is that the crucial difference between the tasks is the importance of processing feedback across a series of trials. In the planning task, each problem is discrete; the feedback on each trial provides relevant information about performance. In the guessing task, feedback to a single trial is less meaningful; it is only when feedback is assimilated across a number of trials that subjects can assess their performance against the level that chance would predict. Further, even though subjects were told to guess at random, they probably monitored performance and chose their responses in the light of previously rewarded responses. Blood flow in the orbitofrontal cortex (see Fig. 3 b), was similar in the 'no feedback' conditions of the two tasks, but was higher in the feedback conditions of the guessing task than the planning task. This suggests that the orbitofrontal activation may be associated with cross-trial processing of feedback information.

Anecdotally people are reported to be irrational in the face of chance-determined events; for example many people would say that if a coin was tossed 10 times, 10 heads would be a less likely outcome than a specified random sequence or that having rolled one six with a dice they are less likely to roll another. Also, people tend to look for patterns in random events (consider the sales figures of books which claim to describe predictable patterns in the outcome of the lottery). These tendencies were confirmed by studying the sequences produced in the guessing task in this study. Only one of the six subjects ever produced the same response on successive trials and when asked to describe how they made their guesses, most subjects produced theories based on spurious patterns. Obviously, a limitation of the design of this study was that subjects were never scanned with feedback given on a 50:50 schedule, as chance would predict. Activations associated with feedback may be different in this situation where the contingencies are less unexpected; this is a question for future research.
The suggestion that ventral orbitofrontal activity is associated with the monitoring of response-outcome relations across a sequence of trials is compatible with the findings of Dias [9] and Rolls [24] that animals and patients with orbitofrontal lesions are impaired on tasks involving reversal learning and extinction. By their very nature, these tasks require cross-trial processing of stimulus -reward relations. The hypothesis is also consistent with recent neurological studies by Damasio and colleagues. Patients with lesions of the orbitofrontal cortex manifest impairments in real-life decision-making in spite of otherwise preserved intellectual abilities [7, 8]. Bechara et al. [4] developed a task to simulate real-life decision-making which typically involves uncertain premises and outcomes together with the processing of reward and punishment. Using this gambling task, these authors found that patients with orbitofrontal lesions are guided by immediate prospects rather than long-term consequences. Even when they can describe the contingencies governing the task, these patients cannot use this knowledge to guide their behaviour, exhibiting a 'myopia for the future'. In a further study [5] this group found that this behavioural effect was accompanied by an absence of anticipatory skin conductance response.

In conclusion, the results of this study suggest that response to feedback involves differential neural activity within a system including regions of the ventral striatum and orbitofrontal cortex; the nature of this neural response depending on the psychological requirements of the task. Differential activation of components of this network as a function of task type may be due to a combination of perceived lack of control over the task and the implicit requirement to process response-outcome relations across a series of trials. These possibilities are not mutually exclusive because in many situations, learning about response-outcome relations is a vital part of determining the extent to which the situation can be controlled. The different patterns of blood flow within the ventral striatal and orbitofrontal regions suggests that these regions fulfil subtly different roles within the network.

This study has potentially important implications for neuropsychiatry. Responses to feedback, success and failure, can be disrupted in psychiatric as well as neurological patients. The phenomena of 'learned helplessness', described above, has been used as an animal model of depression [1]. Behavioural accounts of the disorder have also stressed a crucial role of abnormal responses to social reinforcement in the development and maintenance of depression [20, 21]. In cognitive tasks, depressed patients are less motivated to respond for reward [33]. They also show distorted perceptions when asked to describe the amount of positive reinforcement experienced during performance of tasks [16]. Depression is also associated with oversensitivity to negative feedback $[3,11]$, with subsequent performance likely to be disrupted by perceived failure. The present findings would predict that these abnormalities may be mediated by dysfunction within a 
neural network including regions of the ventral striatum or orbitofrontal cortex.

As Damasio stresses, gambling tasks where outcomes are uncertain and contingencies must be monitored and used to predict future events, can be used as analogues for real-life situations. Identifying the neural substrates of such tasks may prove crucial to our understanding of how people function in an interactive social context. The present findings should be regarded as preliminary evidence and are by no means conclusive. However, the study begins to address this neglected area of functional imaging and is an important step in developing a better understanding of the interface between cognition and emotion.

Acknowledgements--RJD and CDF are supported by the Wellcome Trust.

\section{References}

1. Abramson, L. Y., Seligman, M. E. P. and Teasdale, J. Learned helplessness in humans: Critique and reformulation. Journal of Abnormal Psychology 87, 49-74, 1978.

2. Baker, S. C., Rogers, R. D., Owen, A. M., Frith, C. D., Dolan, R. J., Frackowiak, R. S. J., Robbins, T. W. Neural systems engaged by planning: A PET study of the Tower of London task. Neuropsychologia 6, 515-526, 1996.

3. Beats, B. C., Sahakian, B. J. and Levy, R. Cognitive performance in tests sensitive to frontal lobe dysfunction in the elderly depressed. Psychological Medicine 26, 591-603, 1995.

4. Bechara, A., Damasio, A. R., Damasio, H., Anderson, S. W. Insensitivity to future consequences following damage to human prefrontal cortex. Cognition 50, 7-15, 1994.

5. Bechara, A., Tranel, D., Damasio, H., Damasio, A. R. Failure to respond autonomically to anticipated future outcomes following damage to the prefrontal cortex. Cerebral Cortex 6, 215-225, 1996.

6. Butter, C. M. Perseveration in extinction and in discrimination reversal tasks following selective frontal ablations in Macaca mulatta. Physiology of Behaviour 4, 163-171, 1969.

7. Damasio, A. R., Descartes' Error. Putnam, New York.

8. Damasio, A. R., Tranel, D. and Damasio, H., Somatic markers and the guidance of behaviour. In Frontal Lobe Function and Dysfunction, ed. H. Levin, $\mathrm{H}$. Eisenberg and A. Benton. Oxford University Press, New York, 1991, pp. 217-228.

9. Dias, R., Robbins, T. W., Roberts, A. C. Dissociation in prefrontal cortex of affective and attentional shifts. Nature 380, 69-72, 1996.

10. Dolan, R. J., Fletcher, P., Morris, J., Kapur, N., Deakin, J. F. W. and Frith, C. D., Neural activation during covert processing of happy facial expressions, Neuroimage, (in press), 1996.
11. Elliott, R., Sahakian, Bj., McKay, A. P., Herrod, J. J., Robbins, T. W., Paykel, E. S. Neuropsychological impairments in unipolar depression: The influence of perceived failure on subsequent performance. Psychological Medicine 26, 975-989, 1996.

12. Friston, K. J., Holmes, A. P., Worsley, K. J., Poline, J-B., Frith, C. D., Frackowiak, R. S. J. Statistical parametric maps in functional imaging: A general approach. Human Brain Mapping 2, 189-210, 1995.

13. Gaffan, D. and Harrison, S. Amygdalectomy and dissociation in visual learning for auditory secondary reinforcement by monkeys. Journal of Neuroscience 7, 2285-2292, 1987.

14. Gaffan, E. A., Gaffan, D. and Harrison, S. Disconnection of the amygdala from visual association cortex impairs visual reward association learning in monkeys. Journal of Neuroscience 8, 2285-2292, 1988.

15. George, M. S., Ketter, T. A., Gill, D. S., Haxby, J. V., Ungerleider, L. G., Herscovitch, P., Post, R. M. Brain regions involved in recognizing facial emotion or identity: An oxygen-15 PET study. Journal of Neuropsychiatry and Clinical Neuroscience 5, 384-394, 1993.

16. Hughes, J. R., Pleasants, C. N., Pickens, R. W. Measurement of reinforcement in depression: A pilot study. Journal of Behavioral Therapy and Experimental Psychiatry 16, 231-236, 1985.

17. Jones, B. and Mishkin, M. Limbic lesions and the problem of stimulus-reinforcement associations. Experimental Neurology 36, 362-377, 1972.

18. Iversen, S. and Mishkin, M. Perseverative interference in monkey following selective lesions of the inferior prefrontal convexity. Experimental Brain Research 11, 376-386, 1970.

19. LeDoux, J. E. Emotional memory systems in the brain. Behavioural Brain Research 58, 69-79, 1993.

20. Lewinsohn, P. M., A behavioural approach to depression. In, The Psychology of Depression: Contemporary Theory and Research, ed. R. J. Friedman and M. M. Katz. Winston/Wiley: New York, 1974. pp. 157-185.

21. Lewinsohn, P. M., Youngren, M. A. and Grosscup, S. J., Reinforcement and depression. In The Psychobiology of Depressive Disorders, ed. R. A. Depue. Academic Press, New York, 1979, pp. 291-316.

22. Morris, J. S., Frith, C. D., Perrett, D. I., Rowland, D., Young, A. W., Calder, A. J. and Dolan, R. J., A differential neural response in the human amygdala to fearful and happy facial expressions, Nature, in press.

23. Owen, A. M., Sahakian, B. J., Hodges, J. R., Summers, B. A., Polkey, C. E., Robbins, T. W. Dopamine-dependent frontostriatal planning deficits in early Parkinson's disease. Neuropsychology 9, 126140, 1995.

24. Rolls, E. T., Hornak, J., Wade, D. and McGrath, J. Emotion related learning in patients with social and emotional changes associated with frontal lobe damage. Journal of Neurology, Neurosurgery and Psychiatry 57, 1518-1524, 1994.

25. Russchen, F. T., Bakst, I., Amaral, D. G., Price, J. $\mathrm{L}$. The amygdalostrial projection in the monkey. An 
anterograde tracing study. Brain Research 329, 241257, 1985.

26. Schneider, F., Gur, R. E., Alavi, A., Seligman, M. E., Mozley, L. H., Smith, R. J., Mozley, P. D., Gur, R. C. Cerebral blood flow changes in limbic regions induced by unsolvable anagram tasks. American Journal of Psychiatry 153, 206-212, 1996.

27. Schultz, W., Apicella, P., Scarnati, E. and Ljungberg, $T$. Neuronal activity in monkey ventral striatum related to the expectation of reward. Journal of $\mathrm{Neu}$ roscience 12, 4595-4610, 1992.

28. Selemon, L. D., Goldman-Rakic, P. S. Longitudinal topography and interdigitation of corticostriatal projections in rhesus monkey. Journal of Neuroscience 5, 776-794, 1985.

29. Seligman, M. E. P., Helplessness: on Depression, Development and Death. Freeman, San Francisco, 1975.
30. Shallice, T. Specific impairments in planning. Philosophical Transactions of the Royal Society B298, 199209, 1982.

31. Talairach, J. and Tournoux, P., Coplanar Stereotactic Atlas of the Human Brain. George Thieme Verlag, Stuttgart, 1988.

32. Thorpe, S. J., Rolls, E. T. and Maddison, S. The orbitofrontal cortex: Neuronal activity in the behaving monkey. Experimental Brain Research 49, 93$115,1983$.

33. Wener, A. E., Rehm, L. P. Depressive affect: A test of behavioral hypotheses. Journal of Abnormal Psychology 84, 221-227, 1975.

34. Zajonc, R. B. Feeling and thinking: preferences need no influences. American Psychologist 35, 151-175, 1980. 